\title{
CONTROL AND GAS LEAKAGE DETECTION IN A FINE COAL INJECTION PLANT: DESIGN AND EXPERIMENTS
}

\author{
Wolfgang Birk, Andreas Johansson, and Alexander Medvedev
}

\author{
Control Engineering Group \\ Luleå University of Technology \\ SE - 97187 Luleå, Sweden
}

\begin{abstract}
This paper deals with design and implementation of a combined modelbased control and gas leakage detection system applied to the pulverized coal injection plant at SSAB Tunnplåt AB in Luleå, Sweden. The structure and functions of the in-house control and process monitoring system SafePCI are described. SafePCI is experimentally tested and has successfully completed two weeks test operation. The evaluation of the test operation indicate that combined model-based control and gas leakage detection is a major improvement for control systems in the process industry. Copyright (C) 1998 IFAC
\end{abstract}

Keywords: Model-based control, Fault detection, Pulverized coal injection

\section{INTRODUCTION}

On-line fault detection algorithms require high computational performance, and were, until recently, rather expensive to implement . Nowadays, personal computers reach a performance level and low price that the implementation of model-based control strategies combined with on-line fault detection functions becomes financially attractive for process industry.

\subsection{Process redesign}

The process industry often faces the fact that older plants do not meet the demands for increased production capacity, making improvements necessary either by a new plant purchase or a major reconstruction of the existing plant structure. To avoid high capital investment in equipment and simultaneously gain higher performance, a control system upgrade seems to be a reasonable course of action. Such an upgrade is not very expensive and, usually, gives good results. However, the existing equipment has to operate in a harder working mode which might lead to a higher fault risk. Typically, control valves become subject to an excessive wear after an improved control law is enforced. The resulting leakages in the control valves can cause economical losses and hazards for operational staff. Therefore fault detection and monitoring become necessary. Introducing on-line fault detection in a control system also enables the operators to plan and prepare maintenance stops in advance. Hence, there will be less and shorter maintenance stops.

The Center for Process and System Automation $(\operatorname{ProSA})$ at Luleå University of Technology has established a network comprising four major Swedish process industry companies: AssiDomän, Boliden, LKAB, and SSAB Tunnplåt AB. As a pilot project demonstrating benefits of combined model-based control and fault detection, the existing control system of a Pulverized Coal Injection $(P C I)$ plant is upgraded.

Since coal is $40 \%$ cheaper than coke, injecting pulverized coal instead of using coke is economically beneficial. According to (American Iron and Steel Institute 1998), the share of pulverized coal compared with coke as fuel will rise from $36 \%$ to $50 \%$ by the year 2015 . Improving the performance of an existing PCI plant by upgrading the control 


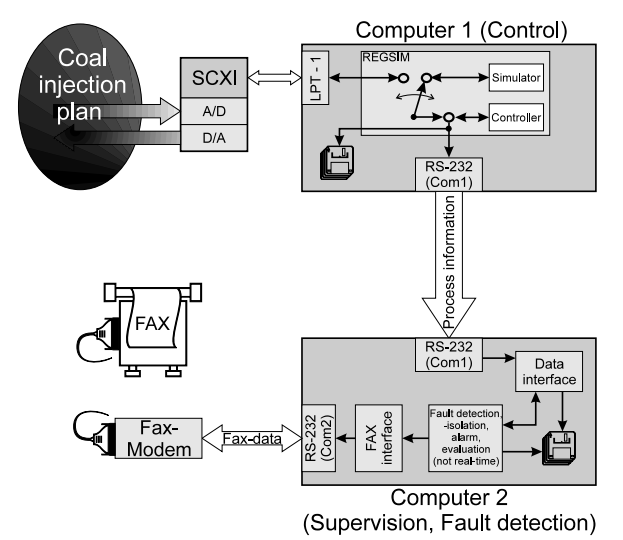

Fig. 3. System structure, hardware and software

Following the hardware structure, the system software can also be divided into two parts: PCIcontrol and PCIguard. PCIcontrol offers three operation modes: real-time, simulation and playback mode. Switching the mode does not effect PCIguard since the transmitted data has mode independent characteristics.

PCIcontrol is a revised version of the software RegSim $^{\circledR}$, (Gustafsson 1995). Communication capability and a driver for the data acquisition device have been added. In PCIguard, not all activities are necessarily real-time, but some of them are event driven. If there are no events like received data, timeouts, messages or operator input through the command-line, the software is running in the stand-by mode. All tasks on this computer run in a time-sharing environment with priorities assigned to each task. The operator has the possibility to change priorities and enable or disable tasks. A special off-line mode makes the software able to run in a stand alone version, as described above.

Fig. 3 depicts the system structure and summarizes the data flow in SafePCI.

\section{PROCESS MODELING}

In order to distinguish between leakages in different valves and control the plant, models describing the process dynamics, including both pressurization and injection, are developed.

First, a non-linear physical model for a simplified vessel structure (Fig. 4) is deriven, for the purpose of gas leakage detection. The non-linear model is then linearized around a working point, yielding a linearized physical model. Finally, a linear model is identified from logged process data, to be used for controller design.

\subsection{Non-linear model}

The non-linear model is based on physical principles and is given by

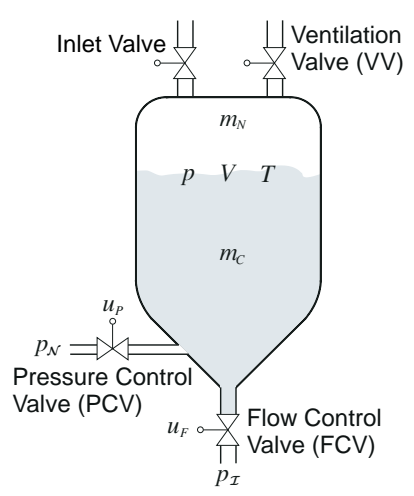

Fig. 4. Schematic drawing of an injection vessel

$$
\begin{aligned}
& \dot{x}=A x+B u \\
& y=h(x)
\end{aligned}
$$

where $A$ and $B$ are constant real matrices obtained by identification. The input vector $u$ is defined by

$$
u \triangleq\left[\begin{array}{l}
f_{\text {liq }}\left(p, p_{\mathcal{I}}\right) g_{F}\left(u_{F}\right) \\
f_{\text {gas }}\left(p, p_{\mathcal{I}}\right) g_{F}\left(u_{F}\right) \\
f_{\text {gas }}\left(p_{\mathcal{N}}, p\right) g_{P}\left(u_{P}\right)
\end{array}\right]
$$

where $g_{F}$ and $g_{P}$ are the characteristic functions of the FCV and PCV (see Fig. 4), respectively and $f_{\text {liq }}$ and $f_{\text {gas }}$ are functions describing the flow of liquid and gas, respectively, over a pressure drop.

The state vector $x=\left[\begin{array}{ll}m_{C} & m_{N}\end{array}\right]^{T}$ represents the masses of coal and nitrogen in the vessel and the output vector $y=[m p]^{T}$ is related to $x$ via the uniquely invertible transformation $h(x)$, (Johansson and Medvedev 1998).

\subsection{Linear models}

As mentioned before, the non-linear model is linearized around a working point. The models validity is restricted to the injection phase, see also (Johansson and Medvedev 1998). The development of the identified linear model is discussed in (Fischer and Medvedev 1998). Using the linearized model, the identified linear model can be validated and the physical nature of the coefficients in the identified model can be revealed.

\section{SYSTEM DESIGN}

The algorithms comprising the system design of SafePCI pertain to following three areas: control, fault detection and isolation, and monitoring. Apart from the monitoring, the adopted solutions are based on the results of former work.

\subsection{Control}

The primary control goals depend on the process phase. 


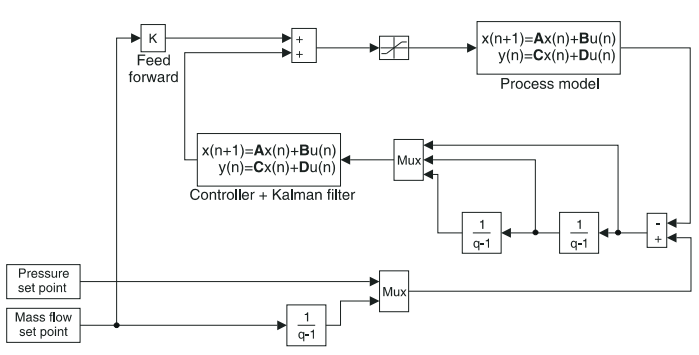

Fig. 5. Block diagram of the MIMO-LQG controller with feed forward

- Pressurization. The pressure has to rise from atmospheric level to the pressure set point for the injection phase. The pressure evolution is described by a ramp, which is the reference signal for the control loop. The controller accuracy is not an issue at this phase.

- Pressure holding. Since the injection vessels are not completely tight, a controller is needed to hold the pressure at set point level during the stand-by.

- Injection. As the primary control goal is to hold the pulverized coal flow to the blast furnace constant, the pressure stability is given less attention. Nevertheless, the goals are to hold the pressure in the vessel at setpoint level and the mass of the injection vessel has to follow a ramp described by the set-point value for the pulverized coal flow.

The controller design is based on the results presented in (Birk and Medvedev 1997). The main difference is not in the controller structure itself, but in the usage of the control scheme. Instead of controlling only one injection vessel, both injection vessels are controlled. Furthermore, the controller is also used during pressurization and pressure holding. Since the controller has been developed basing on the model of one injection vessel, it has to be validated with data from the second injection vessel before being used for both ones. The tests are applied in the same way as presented in (Birk and Medvedev 1997), and have proven that the controller can be used without modification. Effectively a MIMO-LQG controller with feed forward is used, see Fig. 5.

To accomplish the control goals during pressurization and pressure holding, a common controller or two separate controllers can be used for these phases. Since the performance requirements for the pressurization phase are lax, it can be shown that the above controller can be used without modification.

\subsection{Fault detection and isolation}

Three different types of leakages are considered (Table 2). The set of leakages is denoted $\mathcal{L} \triangleq$ $\{\mathcal{A}, \mathcal{N}, \mathcal{I}, \emptyset\}$. A leakage can be interpreted as the flow through a valve with an unknown control signal. The nitrogen leakage flow can thus be represented by

$$
q_{\ell}=k_{\ell} f_{\ell}(\cdot) \quad \ell \in \mathcal{L}
$$

where $k_{\ell}$ is an unknown time-varying factor and $f_{\ell}(\cdot)$ is a function of the pressures on each side of the leakage. The trivial leakage function for the event of 'No Leakage' is $f_{\emptyset}=0$. The other leakage functions $\left(f_{\mathcal{A}}, f_{\mathcal{N}}\right.$ and $\left.f_{\mathcal{I}}\right)$ are developed from the non-linear physical model.

Table 2. Leakages

\begin{tabular}{|l|l|c|}
\hline Leakage & $\begin{array}{l}\text { Possible conse- } \\
\text { quence }\end{array}$ & Notation \\
\hline \hline To the atmosphere & Loss of nitrogen & $\mathcal{A}$ \\
\hline $\begin{array}{l}\text { From the nitrogen } \\
\text { net }\end{array}$ & $\begin{array}{l}\text { Over-pressurized } \\
\text { vessel }\end{array}$ & $\mathcal{N}$ \\
\hline $\begin{array}{l}\text { To/from the injec- } \\
\text { tion pipe }\end{array}$ & Fire & $\mathcal{I}$ \\
\hline No Leakage & - & $\emptyset$ \\
\hline
\end{tabular}

A linear observer for (1) is designed and it is shown that the observer residual is an approximation of the leakage flow $\bar{q}_{L}$ scaled by a constant.

The factor $k_{\ell}$ in (2) is a measure of the size of the hole through which the leakage flow takes place. This means that $k_{\ell}$ varies slowly in time when describing incipient leakages. If $k_{\ell}$ is assumed to be constant during a reasonably long period of time (for example a process cycle), it can be estimated using the Generalized Likelihood Ratio.

Four hypotheses $\left(\mathcal{H}_{\emptyset}, \mathcal{H}_{\mathcal{A}}, \mathcal{H}_{\mathcal{N}}\right.$ and $\left.\mathcal{H}_{\mathcal{I}}\right)$ are formed in agreement with the leakage events. The three leakage hypotheses are tested one by one against $\mathcal{H}_{\emptyset}$ using the Generalized Likelihood Ratio (GLR). If $\mathcal{H}_{\emptyset}$ is rejected in more than one of these tests, the hypothesis with the highest GLR is accepted. The GLR for each leakage hypothesis is

$$
\Lambda_{\ell}\left(\bar{q}_{L}\right)=\frac{\sup _{k_{\ell}>0} P_{\ell}\left(\bar{q}_{L}\right)}{P_{\emptyset}\left(\bar{q}_{L}\right)}
$$

where $P_{\ell}$ is the likelihood function for hypothesis $\mathcal{H}_{\ell}$. The restriction on $k_{\ell}$ comes from the fact that a negative $k_{\ell}$ would imply a leakage flow from a lower pressure to a higher. To complete the fault detection scheme, a threshold for $\Lambda_{\ell}\left(\bar{q}_{L}\right)$ is chosen. When this threshold is exceeded, the null hypothesis is rejected and a leakage has occurred.

See also (Johansson and Medvedev 1998) for more details on the leakage detection scheme.

\subsection{Monitoring}

The monitoring algorithms are a part of PCIguard and have two purposes:

(1) Detection of control system malfunctions

(2) Evaluation of injection phases

SafePCI is a supplement to the existing control system and is therefore not included in the security routines of the latter. Therefore, SafePCI 
needs its own monitoring functions. The following control system malfunctions have to be detected and reacted to:

- Measurement equipment failures

- Crash of the PCIcontrol computer

- Controller wind-up

- Communication malfunction

In all the above cases an alarm message is sent to the operational staff with the diagnosis and a suggested solution to the problem. If the malfunction influences the control, the counter measure is an automatic switch-back to the existing control system. To guarantee such a switch-back, both computers have to send a special formatted signal to the existing control system. PCIcontrol continuously sends a square wave of a specified frequency and PCIguard delivers a specified DC voltage value. If the existing control system does not received one of the signals, it will automatically switch back.

Furthermore, every injection phase is automatically evaluated. The evaluation results are accumulated until a sufficiently high number of injection phases is completed. Then the results are transmitted to the operational staff in a facsimile message. The evaluation tables contain information on:

- Standard deviations in mass and pressure

- Maximum deviations in mass and pressure

- Controller saturation rate

- Mean values of mass and pressure residuals

The injection vessels are represented separately in the table, facilitating comparisons between vessels and trend analyses.

\section{EXPERIMENTS AND TEST OPERATION}

The operation period has been set to two weeks and should be continuous. Therefore, a thorough preparation period with experiments precedes the test operation.

\subsection{Experiments}

Before starting with the test operation, the controllers have to be validated during an experimental run, where the injection vessels are controlled under surveillance for one day. Furthermore, the malfunction scenarios that would not jeapordize plant operation are tested on the plant, whilst the more dangerous faults are simulated. The following tests have been performed on the plant:

- Set point changes

- Control of pressurization and pressure holding

- Switching from one injection vessel to the other

- Process phase independent start-up
Injection vessel S21

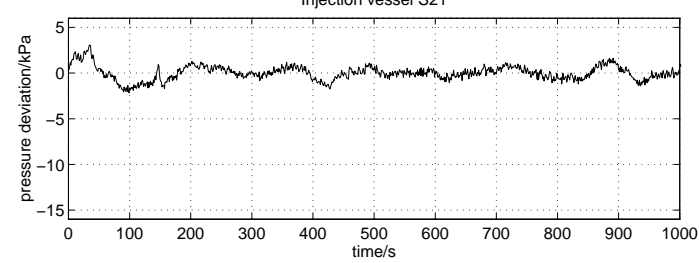

Injection vessel S22

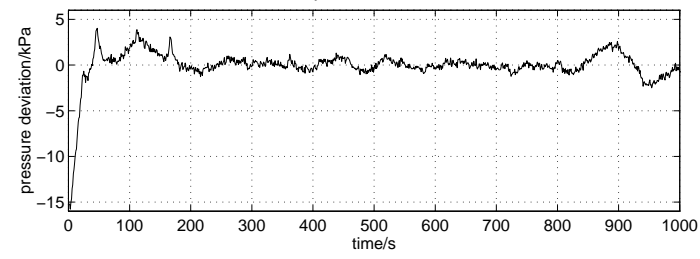

Fig. 6. Pressure deviation for both vessels with the model-based control strategy (Example).

- Measurement equipment malfunction

- Crash of PCIcontrol computer

- Crash of both computers

- Communication malfunction

The leakage detection algorithms are tested in simulation mode, where leakages with a given size can be introduced. There, the following tests have been performed:

- Gas leakage to the atmosphere

- Gas leakage from the nitrogen net

- Gas leakage to the injection pipe

- Several leakages at a time

- Controller performance under existing leakage

All single gas leakages are detected. Only if several leakages appear at the same time, fault detection can not be assured. Regarding the controller performance, the new control strategy tolerates larger leakages and therefore can provide a stable coal flow to the blast furnace notwithstanding gas leakage in the plant.

\subsection{Test operation}

During two weeks, SafePCI had been connected to the coal injection plant and replaced the existing control system throughout nearly 400 injection phases. Fig. 6 and Fig. 7 show pressure and mass deviations, acquired during an injection phase for both vessels. For comparison, Fig. 8 shows the mass and pressure deviation during an injection phase when the injection process is controlled by the existing control system.

In order to compare the existing control strategy with the model-based control strategy, the following performance measures are applied:

- Standard deviation

- Maximum deviation

- Standard deviation of $1^{s t}, 2^{\text {nd }}$ and the last third of an injection phase

- Maximum deviation of $1^{s t}, 2^{\text {nd }}$ and the last third of an injection phase 


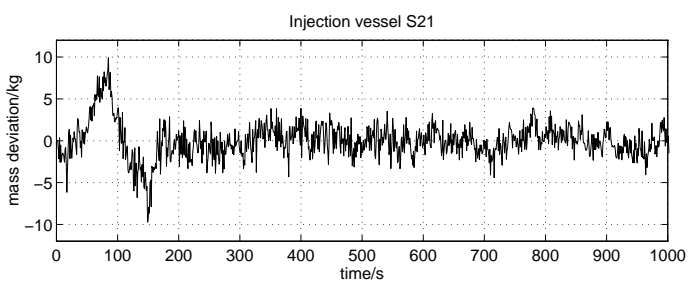

Injection vessel S22

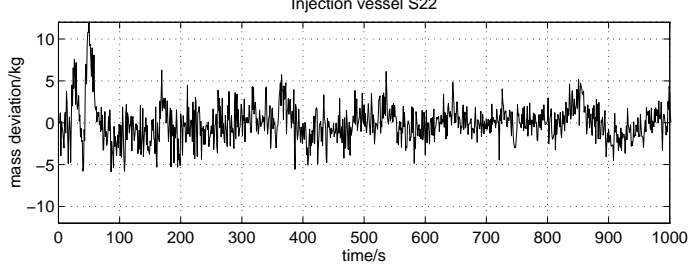

Fig. 7. Mass deviation for both vessels with the model-based control strategy (Example).
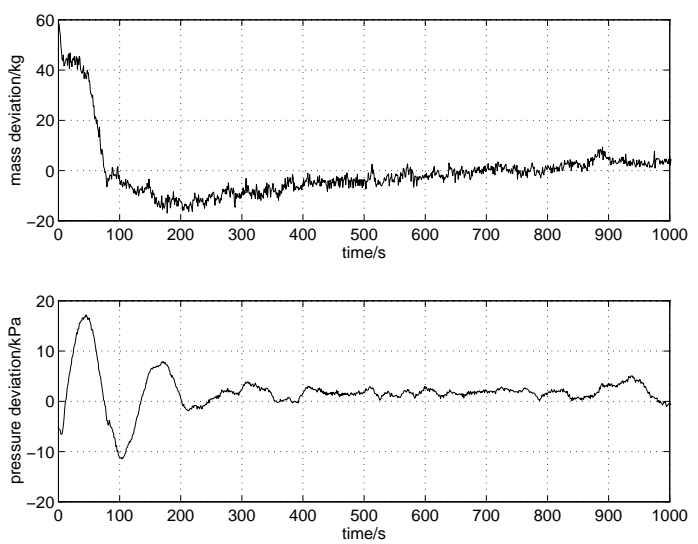

Fig. 8. Mass and pressure deviations with the existing control strategy (Example).

The performance measures are evaluated for the mass and pressure signals during the injection phase and are classified according to which injection vessel has injected. Fig. 9 shows a comparison between the existing and model-based control strategy with respect to standard deviation of the mass. Obviously, the model-based control strategy drastically improves the control performance. The mean values of the performance measures evaluated over all available injection phases are given in Table 3. Notably, although the pressure stabilization has a low priority and in fact is used to facilitate coal flow stabilization, the stabilization of the pressure has been improved, too.

Table 3. Improvements

\begin{tabular}{|c|c|c|}
\hline Measure & Pressure & Mass \\
\hline \hline Standard deviation & $45.6 \%$ & $82.5 \%$ \\
\hline Maximum deviation & $20.2 \%$ & $79.8 \%$ \\
\hline
\end{tabular}

Concerning the leakage detection, no leakage has been detected during the test operation and an examination of the plant showed that no visible leakages occurred. Hence, no false alarm has been generated, what is a positive result. Putting this together with the results from the experiments, the gas leakage detection is proven to work well.

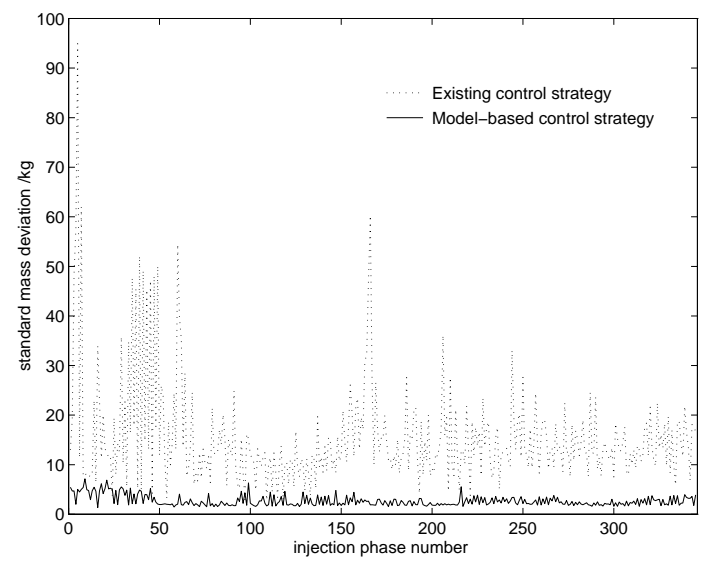

Fig. 9. Standard deviation of the mass for the model-based and the existing control strategy

\section{CONCLUSIONS}

The design and the implementation of a combined control and gas leakage detection system are discussed. Experiments and two weeks long test operation have been carried out at the actual plant. The positive effects expected from simulation and short term experiments are confirmed by the test operation results. Introducing modelbased control strategies combined with on-line fault detection function improves not only the control performance, but as well facilitates plant maintenance and security. More advanced faulttolerant control strategies can take advantage of the on-line fault detection functions, so that control performance in the presence of malfunction can be maximized. Hence, the pulverized coal flow to the blast furnace can be maximized, and the costs for iron production be reduced.

\section{REFERENCES}

American Iron and Steel Institute (1998). Steel industry technology roadmap. At http://www.steel.org/MandT/contents.htm.

Birk, W. and A. Medvedev (1997). Pressure and flow control of a pulverized coal injection vessel. In: Proceedings of the 1997 IEEE International Conference on Control Applications. pp. 127-132.

Fischer, B. and A. Medvedev (1998). Laguerre shift identification of a pressurized process. To be presented at the American Control Conference in Philadelphia, June 1998.

Gustafsson, T. (1995). Regsim: A software tool for real time control and simulation. In: Proc of the 4th IEEE Conference on Control Applications, Albany, New York 1995.

Johansson, A. and A. Medvedev (1998). Modelbased leakage detection in a pulverized coal injection vessel. To be presented at the American Control Conference in Philadelphia, June 1998. 